This is a post-peer-review, pre-copyedit version of an article published in Psychological Research. The final authenticated version is available online at: http://dx.doi.org/10.1007/ s00426-015-0719-0".

\title{
Physiological threat responses influence number processing
}

\author{
Scholla *, A., Moeller $^{\mathrm{a}, \mathrm{b}}$ *, K., Scheepers ${ }^{\mathrm{c}}$, D. T., Nuerk ${ }^{\mathrm{a}, \mathrm{b}}$, H.-C., \& Sassenberg a, b, K. \\ ${ }^{\text {a }}$ Knowledge Media Research Center, Schleichstr. 6, 72076 Tuebingen, Germany \\ ${ }^{\mathrm{b}}$ Department of Psychology and LEAD Graduate School, University of Tuebingen, Schleichstr.4, \\ 72076 Tuebingen, Germany \\ ${ }^{\text {c }}$ Department of Psychology, Leiden University, P.O. Box 9555, 2300 RB Leiden, The Netherlands \\ * the first two authors contributed equally to this work
}

Please address correspondence to:

Annika Scholl

Knowledge Media Research Center

Schleichstr.6

72072 Tuebingen, Germany

Fon: +49 7071979257

Email: a.scholl@iwm-kmrc.de 


\begin{abstract}
Being able to process numbers - such as solving a math task or roughly comparing product prices - is a key competency. Yet, previous research suggests that negative affective responses, such as math anxiety or experienced threat towards numbers, reduce performance. However, such research mainly relied on self-report measures. The present research sought to go one step further by investigating how physiological responses indicative of threat predict math performance. Individuals' physiological responses while solving a task reflect whether they evaluate the task as exceeding or matching their personal resources (i.e., respond to the task as a threat vs. challenge), and these responses in turn predict subsequent performance. We thus assumed that the more individuals exhibit a threat (rather than challenge) physiological response to a numerical task, the worse they would perform on this task. Results of an experiment with cardiovascular indicators of threat vs. challenge (i.e., measuring the efficiency of blood transportation) supported this assumption. The findings thereby contribute to an understanding of how threat responses to numerical tasks can hinder performance and provide first indications how such performance decrements might be mitigated.
\end{abstract}

(181 words)

Keywords:

numeracy, math performance, threat, biopsychosocial model, cardiovascular measures 


\section{INTRODUCTION}

Numeracy - the ability to appropriately deal with and handle numbers - is a key competency for our life, with deficits causing severe consequences on both the individual (e.g., Parsons \& Bynner, 2005), but also the societal level (Gross, Hudson, \& Price, 2009). However, there is accumulating evidence from different lines of research indicating that performance on numerical tasks is influenced reliably by situational and individual factors. On the one hand, recent research clearly indicates influences of task characteristics on number processing, such as item sequence (i.e., the Gratton effect; Gratton, Coles, \& Donchin, 1992; see Macizo \& Herrera, 2011; Huber, Moeller, Nuerk, Macizo, Herrera, \& Willmes, 2013 for a Gratton effect in number processing) or the relative frequency of item categories (i.e., proportion congruity effect; Tzelgov, Henik, \& Berger, 1992; see Macizo \& Herrera, 2012, for a proportion congruity effect in number processing). On the other hand, there is a comprehensive literature from educational research indicating that negative affective responses (e.g., anxiety) towards mathematics guide performance in numerical tasks and mathematics achievement in general (e.g., Moenikia \& Zahed-Babelan, 2010; Else-Quest, Hyde, \& Linn, 2010). In this work it has been suggested that math anxiety exhibits the most detrimental influence on mathematics achievement (e.g., Eden, Heine, \& Jacobs, 2013; Maloney \& Beilock, 2012, for reviews, but see, e.g., Krinzinger, Kaufmann, \& Willmes, 2009, for diverging data).

Interestingly, research going beyond self-reported negative affective responses towards numbers (e.g., Beilock, 2008; Beilock \& Carr, 2005, see also Eden et al., 2013, for the case of math anxiety) and trying to evaluate the mechanisms underlying (potentially) worse performance is rather sparse. The present research aims at pursuing this issue by considering physiological responses to numbers (i.e., threat responses). Integrating research on threat / challenge responses (Blascovich \& Tomaka, 1996) and numerical cognition, we argue that numerical performance is associated with individuals' physiological responses to a numerical task (i.e., their cardiovascular reactivity), with these physiological responses reflecting how individuals (affectively and cognitively) evaluate the task. When a given task is seen as threatening (i.e., exceeding individuals' perceived personal abilities) rather than challenging (i.e., demanding but manageable with their perceived abilities), this is reflected by specific physiological responses indicating threat and challenge motivational states. When 
measured appropriately, these physiological indicators should predict performance on the task at hand (for an overview see Moore, Vine, Wilson, \& Freeman, 2012). We thus assumed that physiological threat / challenge responses to a numerical task should be associated with performance on this task. The present research investigated this idea by means of well-established physiological measures that have repeatedly been linked to performance in other domains (e.g., sports, decision making, etc.; Blascovich, Seery, Mugridge, Norris, \& Weisbuch, 2004; De Wit, Scheepers, \& Jehn, 2012; Seery, Weisbuch, Hetenyi, \& Blascovich, 2010).

\section{How negative affective responses harm performance}

The majority of studies investigating how negative affective responses account for decreasing performance assume that cognitive resources important for task performance are distracted away from the task solution, in order to regulate the respective affective response. Nùñez-Peñs and Colomé (2014) termed this "a reactive and compensatory recruitment of control resources" (p. 1) to deal with negative affect. In this domain, Attentional Control Theory (Eysenck, Derakshan, Santos, \& Calvo, 2007), as one of the main theoretical approaches, attributes the negative consequences of negative affective responses for cognitive performance to impaired functioning of the central executive, as proposed in the working memory model by Baddeley (1986). Negative affective responses (e.g., anxiety, worrying thoughts and ruminations; Eysenck et al., 2007) impair top-down goal-driven behaviour, which in turn increases the reliance on bottom-up stimulus driven behaviour and, ultimately, leads to higher distractability. A reactive recruitment of additional cognitive resources to compensate for intrusions of task-irrelevant information and thoughts then leads to reduced task performance (for a more detailed discussion of these processes see Derakshan \& Eysenck, 2009; see Ansari \& Derakshan, 2011 for neural correlates).

Importantly, this explanation has been provided for a wide range of different situations and populations, such as "choking under pressure" and performance deficits associated with math anxiety (e.g., Beilock, 2008; Ramirez Gunderson, Levine, \& Beilock, 2013). For instance, Ashcraft and Kirk (2001) concluded that at least part of the poor performance of individuals showing negative affective 
responses towards math stems from an anxiety-related depletion of general cognitive resources, in particular working memory capacity (see also Beilock \& Carr, 2005).

In short, these approaches suggest that negative affective responses consume cognitive resources, which are then no longer available for effectively solving the task at hand. As a result, negative affective responses considerably undermine performance (e.g., Eden et al., 2013; Maloney \& Beilock, 2012, for reviews). This effect is especially pronounced when the task is difficult (i.e., cognitively demanding; e.g., Ashcraft, 2002; Cates \& Rhymer, 2003; Hopko, Ashcraft, Gute, Ruggiero, \& Lewis, 1998; Hopko, McNeil, Lejuez, Ashcraft, Eifert, \& Riel, 2003; see Ashcraft, 2002, for a review). Notably, this notion closely relates to a more specific case of negative affective responses, namely threat and challenge responses. More specifically, physiological threat responses to a task similarly predict performance in different domains of (cognitive) performance (e.g., Blascovich et al., 2004; De Wit et al., 2012; Moore et al., 2012; Seery et al., 2010). However, these threat and challenge indicators have not yet been applied to the domain of numerical performance.

\section{The role of physiological threat / challenge responses for performance}

Solving a numerical task represents a situation in which individuals should be motivated to perform well and need to invest instrumental cognitive resources. Such motivated performance situations cause individuals to evaluate whether or not they will be able to cope with the situation. This is described in the biopsychosocial model of challenge and threat (BPSM; Blascovich, 2008; , \& Mendes, 2010; Blascovich \& Tomaka, 1996).

According to this model, during motivated performance individuals weigh the perceived situational demands (e.g., effort the task requires, uncertainty) against their perceived personal resources (e.g., knowledge, skills, support), resulting in a threat or challenge response: When situational demands exceed perceived personal resources ("I feel that my resources do not suffice"), threat occurs. When situational demands match up or fall below perceived personal resources ("I feel I can master this"), challenge occurs (Blascovich \& Mendes, 2010; Blascovich \& Tomaka, 1996; Tomaka, Blascovich, Kelsey, \& Leitten, 1993). As such, threat / challenge responses can be seen as 
motivational states along one continuum, with challenge being associated with a relatively better availability of cognitive resources and better performance than threat (Blascovich, 2008).

Importantly, this demands-against-resources evaluation takes place on the affective and cognitive level, and is not necessarily factually correct or conscious (Seery et al., 2010; WeisbuchRemington, Mendes, Seery, \& Blascovich, 2005). In other words, challenge and threat are subjective evaluations, and individuals are often not able to accurately report such inner states (e.g., Nisbett \& Wilson, 1977). In addition, drawing individuals' attention to their threat / challenge reaction, for instance via conventional self-report assessment, might in itself alter the actual threat / challenge response (Seery et al., 2010) or result in defensive responses in case of current threat (Blascovich, 2000). This renders more direct indicators of threat (and challenge), which can be measured online and unobtrusively, important.

Therefore, we considered well-established physiological indicators of threat / challenge (for an overview see Blascovich, 2008). As such, threat / challenge responses are evident in distinct patterns of cardiovascular reactivity, which reflect the efficiency of the heart in blood transportation (i.e., energy mobilization; Blascovich, 2008; Blascovich \& Mendes, 2010; Blascovich \& Tomaka, 1996). In this context, two main indicators of cardiovascular reactivity are crucial: Vascular resistance (total peripheral resistance, $T P R$ ), as an index of net constriction versus dilation in the arterial system, and cardiac performance (cardiac output, $\mathrm{CO}$ ), as the amount of blood pumped by the heart. Relatively low TPR and high CO indicate challenge - in other words, blood transportation is highly efficient. In contrast, high TPR and low (increases in) CO reflect threat - that is, a less efficient cardiovascular response to a task. Taken together, challenge is indicated by relatively higher CO and lower TPR than threat (e.g., Seery et al., 2010), which predicts subsequent task performance (e.g., Blascovich et al., 2004; De Wit et al., 2012; Seery et al., 2010). The present research aimed at extending this to the domain of numerical performance.

\section{The present research}

In the present study, we investigated whether physiological threat / challenge responses predict numerical performance. To do so, we examined the relation between cardiovascular responses 
to a numerical task and actual task performance on a number bisection task (NBT, Nuerk, Geppert, van Herten, \& Willmes, 2002).

In the number bisection task, participants have to indicate whether the central number of a triplet reflects the arithmetic mean of the interval, defined by the two outer numbers, or not (e.g., correctly bisected triplet: $4 \_7 \_10$ vs. incorrectly bisected triplet: $3 \_7 \_10$, respectively). We expected that the more physiological responses indicate individuals to experience the task as challenging (rather than threatening) the higher their performance should be.

Importantly, this association between physiological response and performance should be influenced by task difficulty: the association should be stronger for more difficult conditions that require substantial cognitive resources (cf. Blascovich \& Mendes, 2000): Physiological responses indicating the task to be challenging rather than threatening imply that more individual (cognitive) resources are available for task completion. Indeed, Blascovich, Mendes, Hunter, and Salomon (1999) demonstrated that threat versus challenge only predicted performance on difficult tasks (i.e., those tasks that were not well-learned versus those that were well-learned). In other words, a challenge versus threat state only alters performance, if the task at hand requires substantial cognitive resources, rather than being solvable by applying mere heuristics. Importantly, this prediction is corroborated by Attentional Control Theory (e.g., Eysenck et al., 2007), as described above: The detrimental influence of negative affective responses should be more pronounced for difficult tasks (for a review see Ashcraft, 2002). Therefore, we varied task difficulty in the present study.

In line with previous studies using the verification version of the NBT (Nuerk et al., 2002; Moeller et al., 2009; Wood et al., 2008), we manipulated task difficulty independently for correctly and incorrectly bisected triplets. For correctly bisected triplets, we discerned triplets spanning an either small or wide numerical range (e.g., 2_5_8 with a small range of 6 vs. 2_9_16 with a wide range of 14) - with the latter being more difficult to solve (e.g., Nuerk et al., 2002). On the other hand, triplets with either a large or small numerical distance between the central number of the triplet and the correct arithmetical mean of the triplet were used [e.g., 2_4_14 with a large distance (4) to the correct mean (8) vs. 2 _ _ 14 with a small distance (1) to the mean (8)]. Here, incorrectly bisected triplets with a small distance to the mean are more difficult (e.g., Nuerk et al., 2002). 
Accordingly, we expected that for correctly bisected triplets, the positive association between a challenge (rather than threat) response and performance should be more pronounced for triplets with a wide bisection range, whereas for incorrectly bisected triplets, we expected the association to be more pronounced for triplets with a small distance to the arithmetic mean.

\section{METHOD}

Participants. Sixteen undergraduate students (94\% female; Age: $M=20$, Range 18-24) at a Dutch university participated in this and another (unrelated) study in exchange for five Euro or course credit. Informed consent was obtained from all individual participants included in the study. ${ }^{1}$

Task, Stimuli, and Measures. Number bisection task. Participants completed a verification version of the number bisection task, in which they indicated for each trial whether a given number triplet is bisected correctly (i.e., the central number reflects the arithmetical mean of the respective numerical interval, e.g., 23_26_29) or incorrectly by the central number (e.g., 23_25_29). For a total of 100 triplets, this bisection status had to be evaluated with half of the triplets requiring a "yes" and the other half requiring a "no" response. In the current study, we only used the non-multiplicative correctly bisected triplets and incorrectly bisected triplets for which an integer mean exists (from the set by Moeller et al., 2009, Appendix B1). For correctly bisected triplets, bisection range was manipulated (small: 4-8, e.g., 22_24_26 vs. wide: 12-18, e.g., 22_31_40), whereas the distance of the central number of the triplet to the correct mean of the triplet was manipulated for incorrectly bisected triplets (far: 2-8, e.g., 20_21_28 vs. near: 1, e.g., 20_23_28).

Performance accuracy, this means the number of incorrectly classified triplets (i.e., classification errors) served as dependent variable. As can be read from Appendix A in Moeller et al. (2009), numerical attributes of the stimuli (e.g., sum of the three numbers, decade crossings, etc.) were matched between item categories as far as possible.

Physiological indicators for threat and challenge. The primary measures comprised the cardiovascular reactivity during the number bisection task. Apart from CO and TPR, we also assessed Heart Rate (HR; beats per minute) and Pre-Ejection Period (PEP; indicating left-ventricular contractile force). Although PEP and HR reactivity do not distinguish between challenge and threat, a shorter PEP and increased HR is indicative of task engagement, a core aspect of motivated 
performance. Therefore, in research applying the biopsychosocial model, reactivity in PEP and HR is reported as a check on whether task engagement is present (i.e., whether PEP and HR change from the actual task compared to the baseline; Blascovich et al., 2004; De Wit et al., 2012; Seery et al., 2010). ${ }^{2}$

Procedure. Participants, up to two at a time, were invited to take part in a study involving a number bisection task. They received all instructions on a computer screen. Physiological measurements were assessed during the complete study. First, the experimenter applied sensors for cardiovascular (CV) recording to each participant. We recorded physiological measures noninvasively according to psychophysiological guidelines (Sherwood et al., 1990). A Biopac MP150 system (Biopac Systems In., Goleta, CA) continuously assessed impedance-cardiographic (ICG) and electrocardiographic signals (ECG). In addition, blood pressure was continuously measured using a Nexfin ${ }^{\circledR}$ BMEYE blood pressure monitor, which makes use of a finger cuff that was attached to the participant's non-dominant hand. The data was stored using the Acqknowledge software (Biopac Systems Inc., Goleta, CA) and the ICG scored using AMS-IMP software (Vrije Universiteit, Amsterdam, the Netherlands).

Baseline measures were recorded for five minutes, during which participants sat for a quiet period and watched a short movie. This was done as to enable comparisons between participants' cardiovascular performance in the baseline versus while they performed the numerical task.

Participants then received instructions for the number bisection task. To emphasize speed, task instructions indicated that each task had to be solved within 5 seconds. Participants solved 10 practice trials and reported their perceived resources and demands with regard to the task before proceeding to the actual task.

\section{RESULTS}

\section{Influence of bisection range and distance to the correct mean}

First, we evaluated whether performance was influenced by the manipulated factors bisection range for correctly bisected triplets and distance between the central number and the correct arithmetic mean of the triplet for incorrectly bisected triplets using paired $t$-tests. 
In line with the results of previous studies using the NBT (e.g., Nuerk et al., 2002), the $t$-tests indicated that participants committed significantly more classification errors for correctly bisected triplets spanning a wide range as compared to triplets spanning a small range on average [5.25 vs. 3.13 errors, respectively, $t(15)=2.96, p<.01]$. Moreover, as expected, incorrectly bisected triplets with a small distance to the arithmetic mean were on average classified more erroneously than triplets with a large distance to the arithmetic mean [3.69 vs. 1.44 classification errors, respectively, $t(15)=3.22, p<$ $.01]$. These results indicate that the manipulation of difficulty for correctly and incorrectly bisected triplets was successful.

\section{Preliminary analyses of cardiovascular reactivity.}

Following standard practice, we focused for all cardiovascular indicators on mean levels during the last minute of the baseline and the first minute of the number bisection task. Reactivity scores were created for HR, PEP, CO, and TPR by subtracting baseline scores from task scores (de Wit et al., 2012; Scheepers et al., 2012; Seery et al., 2010). Reactivity scores were checked for outliers (i.e., values more than $3.3 S D$ s above/below the mean). Outliers were replaced with the value of the next most extreme score (Weisbuch-Remington et al., 2005).

In addition to examining $\mathrm{CO}$ reactivity and TPR reactivity as indicators of challenge and threat separately (see below), we also calculated a combined threat-challenge index (TCI) by subtracting TPR reactivity scores from $\mathrm{CO}$ reactivity scores (both $z$-transformed). Higher values on the resulting index thus signal a relatively higher challenge response. Advantages of using the TCI are that it represents relative threat/challenge tendencies in a single and more reliable index (Seery et al., 2010).

As indicated above, a prerequisite for challenge / threat is task engagement, as indicated by an increase in HR and a decrease in PEP (for the task, relative to baseline levels). While PEP decreased significantly from baseline (i.e., zero; $M=-4.75, S D=5.31$ ), $t(15)=-3.58, p=.003$, HR did not change from baseline levels, $t(15)=-0.78, n s .^{3}$

Main analyses: How cardiovascular indicators predict performance. 
Following our hypothesis, a physiological challenge response should be associated with better performance - especially for the more difficult conditions of correctly and incorrectly bisected triplets requiring more cognitive resources.

A correlation analysis revealed that the threat-challenge index was indeed negatively related to the number classification errors for both correctly bisected triplets with a small $[r(16)=-.47, p<.05$, tested one-sided $]$ and wide bisection range $[r(16)=-.55, p<.05$, tested two-sided, see Fig. 1 and Table 1]. This indicates that the more individuals demonstrated challenge rather than threat responses to the task, the less classification errors they committed. However, the Williams-Steiger Z-test did not confirm our hypothesis that the association between the threat-challenge index and classification performance differed between triplets with a small and large bisection range $[Z=0.56, p=.58] .{ }^{4}$

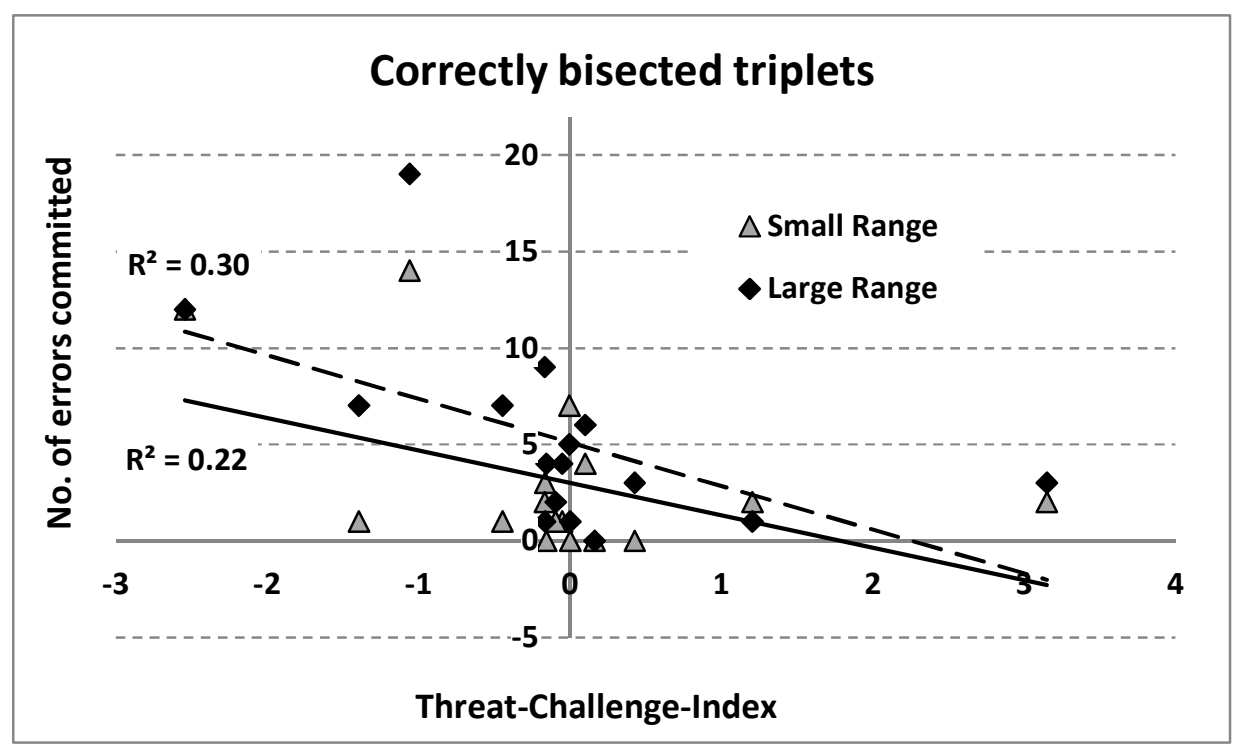

Fig. 1: Correlation of threat-challenge-index and the number of errors committed for correctly bisected triplets with large (dashed line) and small (full line) range

For incorrectly bisected triplets, the correlation analysis indicated that classification performance was neither related reliably to the threat-challenge index for triplets with a small distance to the arithmetic mean $[r(16)=.08, p=.78]$ nor for triplets with a large distance to the arithmetic mean $[r(16)=-.28, p=.30$, see Fig. 2 and Table 1]. Again, the Williams-Steiger Z-test did not indicate a significant difference between the association of the threat-challenge index and 
classification performance for triplets with a small and large distance to the arithmetic mean $[Z=1.17$, $p=.24]$

The above-reported pattern of results on the combined threat-challenge index, seemed mainly driven by variations in $T P R$, indicating that the higher participants' TPR reactivity was, the more classification errors they committed on both correctly bisected triplets with a small $[r(16)=.53, p<$ $.05]$ and wide bisection range $[r(16)=.63, p<.01$, see Table 1]. Again, this was not the case for incorrectly bisected trials [small distance to the arithmetic mean: $r(16)=.12, p=.65$; large distance to the arithmetic mean: $r(16)=.39, p=.13]$.

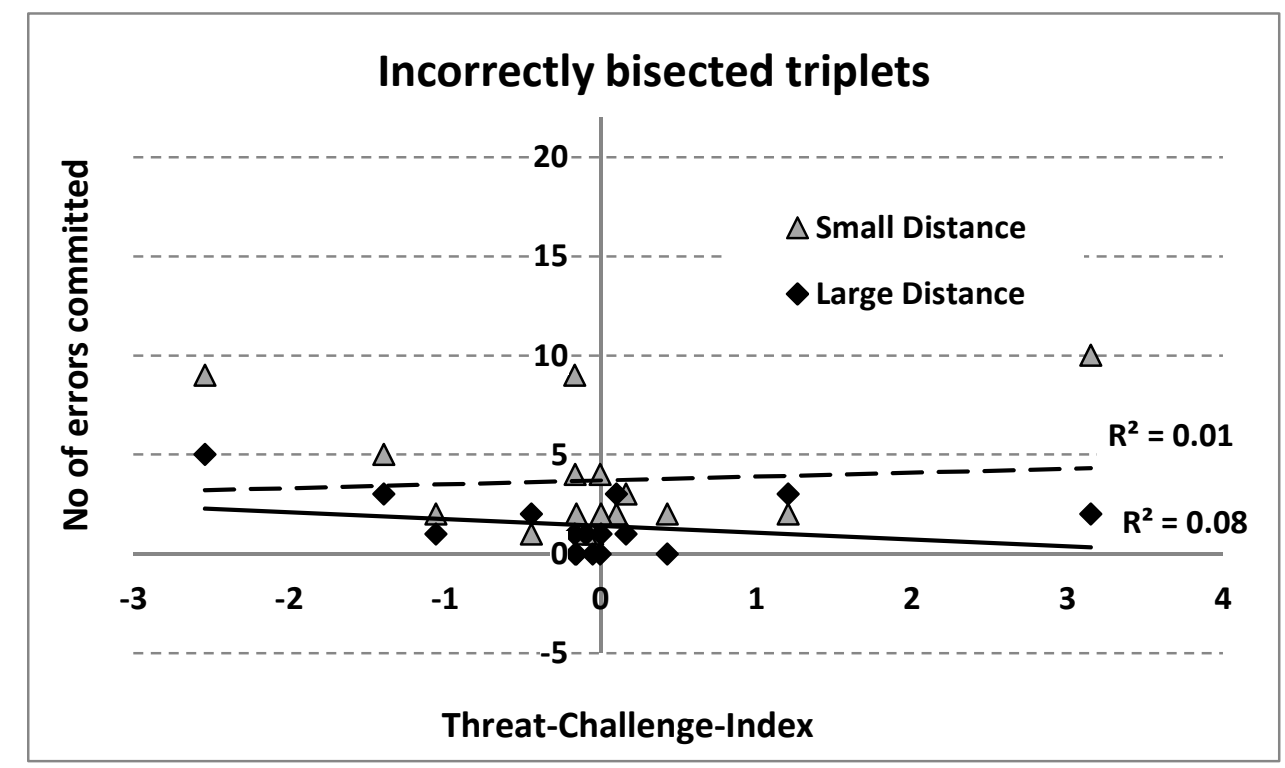

Fig. 2: Correlation of threat-challenge-index and the number of errors committed for incorrectly bisected triplets with small (dashed line) and large (full line) distance between the central number of the triplet and its actual arithmetic mean.

In contrast, the relation between performance and the threat-challenge index did not seem to be driven by changes in $C O$, which was not significantly related to the number of classification errors for correctly bisected triplets [small range: $r(16)=-.29, p=.28$; wide range: $r(16)=-.34, p=.20$ ] and incorrectly bisected triplets [small distance to the arithmetic mean: $r(16)=.36, p=.17$; large distance to the arithmetic mean: $r(16)=-.06, p=.83$, see Table 1$]$. 
This indicates that on the physiological level, a decrease of TPR (i.e., vasodilation), more so than an increase in CO (i.e., cardiac performance) was related to better performance on correctly bisected triplets.

Table 1: Overview of associations of physiological indicators and performance (i.e., number of errors) in the NBT

\begin{tabular}{lcccccc}
\hline & TCI & TPR & CO & CBSR & CBLR & IBSD \\
\hline Threat-Challenge-Index (TCI) & & & & & & \\
\hline Total peripheral resistance (TPR) & $-.95^{* *}$ & & & & & \\
\hline Cardiac output (CO) & $.89^{* *}$ & $-.71^{* *}$ & & & & \\
\hline Correctly bisected small range (CBSR) & $-.47^{\circ}$ & $.53^{*}$ & -.29 & & & \\
\hline Correctly bisected large range (CBLR) & $-.55^{*}$ & $.63^{* *}$ & -.34 & $.81^{* *}$ & & \\
\hline Incorrectly bisected small distance (IBSD) & .08 & .12 & .36 & .26 & .22 & \\
\hline Incorrectly bisected large distance (IBLD) & -.28 & .39 & -.06 & .34 & .30 & .40 \\
\hline
\end{tabular}

Note. ${ }^{* *} \mathrm{p}<.01, * \mathrm{p}<.05,{ }^{\circ} \mathrm{p}<.05$ tested one-sided

\section{DISCUSSION}

Solving a numerical task requires cognitive resources and constitutes a situation in which individuals are usually motivated to perform well - but, nonetheless, sometimes fail at. Previous research indicates that not only characteristics of the task reliably predict numerical performance (e.g., carry addition problems are more difficult than non-carry problems, such as $19+17=36$ vs. $12+24=$ 36). Additionally, individual affective responses towards numbers also play a crucial role (e.g., Eden, Heine \& Jacobs, 2013 for a review). Extending prior studies on self-reported affect or anxiety towards numerical tasks (e.g., Maloney \& Beilock, 2012 for a review), the present research focused on whether physiological indicators of such affective responses - namely, threat / challenge responses as proposed by the biopsychosocial model (e.g., Blascovich, 2008; Blascovich \& Mendes, 2010; Blascovich \& Tomaka, 1996) are predictive of numerical performance. In particular, we hypothesized that a physiological response pattern indicative of challenge (rather than threat) should be associated with 
better performance in a number bisection task. In addition, we assumed this relation to be more pronounced for cognitively demanding (i.e., more rather than less difficult) conditions.

The current results corroborated this hypothesis at least partially. In line with our expectations, we observed that the more individuals' physiological response indicated challenge rather than threat regarding the numerical task, the better their performance was (i.e., the less errors they committed). Thereby, these results extend previous research on how processes on the individual level predict math performance. In particular, we provide new evidence suggesting that apart from self-reported measures (e.g., self-reported math anxiety), physiological indicators differentiating threat vs. challenge responses (based on an appraisal of personal resources and situational demands) allow predicting performance in a numerical task.

In this regard, our research integrated findings on the influence of negative affective responses towards numerical tasks with findings on physiological threat / challenge responses. The present results substantiate previous results on how self-reported negative affective responses deter math performance (e.g., Eden, Heine \& Jacobs, 2013 for a review) by revealing a similar association with threat / challenge - that is, with more direct physiological measures that are indicative of specific motivational states (rather than more general affective reactions to a task). As a next step, it would be desirable to have future studies extending the present approach to further evaluate in how far selfreport measures (e.g., reported math anxiety) are correlated with these physiological indicators of threat / challenge. Additionally, it needs to be clarified whether the physiological indicators introduced in this study indeed complement and extend earlier findings on self-report measures by proving to be predictive over and beyond the latter.

Moreover, applying the propositions of the BPSM (Blascovich, 2008; Blascovich \& Mendes, 2010; Blascovich \& Tomaka, 1996) regarding physiological responses to threat / challenge to the domain of numerical performance allows for more specific assumptions on how negative affective responses may arise (and potentially be compensated for): threat / challenge responses are argued to be a result of the subjective evaluation of personal resources (e.g., one's own skills) against task demands (i.e., what the task requires). One may thus conclude that individuals responding with threat (rather than challenge) to numerical tasks may potentially overweigh the task demands and / or underestimate 
their personal skills. A possible process by which the challenge cardiovascular pattern might be related to better performance is more focused attention to task-relevant cues. Previous work has indeed linked challenge to more task-relevant attention, while threatened people may in addition also focus their attention on task-irrelevant things (e.g., "rumination"; Moore et al., 2012). Interestingly, an explanation in these terms is also in line with previous work showing the importance of attentional processes, such as shifting and focusing, for performing the NBT (Moeller, Fischer, Nuerk, \& Willmes, 2009). Moreover, this is also in line with Attentional Control Theory associating math anxiety with performance decrements due to reduced attentional control (see Ashcraft, 2002, for a review).

As such, the current work substantiates the attention explanation for performance on the NBT not only indirectly, but also provides further insight into the type of tasks on which performance can be predicted by cardiovascular challenge / threat responses. Further research using this paradigm would be desirable to substantiate these claims and evaluate approaches to influence individual appraisal processes.

However, having said this, two important points need to be considered. On the one hand, the present data did not support our more specific hypotheses predicting that above described association of threat / challenge and task performance should be more pronounced for the more difficult task conditions (i.e., for correctly bisected triplets with a large vs. a small range; e.g., 23_29_35 vs. 27_29_31; and the incorrectly bisected triplets with a small vs. large distance to the correct mean; e.g., $23 \_30 \_35$ vs. 23_24_35). On the other hand, we only observed this association consistently for "yes" responses to correctly bisected triplets, but not for "no" responses to incorrectly bisected triplets. Taken together, this seems to imply that there may be a systematic difference between correctly and incorrectly bisected triplets, which overrode the intended difficulty manipulation by numerical magnitude characteristics.

When constructing the stimuli, we hypothesized that actual demands on the processing of number magnitude should be higher for correctly bisected triplets spanning a large range, as these require operating upon wider numerical ranges on the mental number line, and for incorrectly bisected triplets with a small distance to the middle, as these require more accurate localization of numbers on 
the mental number line. However, the present data suggest that processing correctly bisected triplets was more demanding in general. Importantly, apart from the reliable association with the physiological responses, this is corroborated by a directed evaluation of this argument by means of participants' response times. These were reliably longer following correctly as compared to incorrectly bisected triplets (2635ms vs. $2529 \mathrm{~ms}$, respectively; $t(15)=1.94, p<.05$, tested one-sided).

Interestingly, closer inspection of the stimuli revealed that apart from the manipulation of magnitude-related characteristics within both correctly and incorrectly bisected triplets, there also seemed to be another more relevant difference between correctly and incorrectly bisected triplet driving the present results. In 13 of the 50 incorrectly bisected triplets, sequences of adjacent numbers were included (e.g., 21_22_30 or 21_29_30) and in another 17 incorrectly bisected triplets, sequences in steps of two were involved (e.g., 21_23_30 or 21_28_30). In contrast, there were no correctly bisected triplets in which directly adjacent numbers were used (e.g., 21_22_23) and only 3 correctly bisected triplets representing a sequence of steps of two (e.g., 21_23_25). Thus, participants may have adapted to those characteristics and used sequences of adjacent numbers or sequences of steps of two as a heuristic cue to easily detect incorrectly bisected triplets, which required a "no" response. As such a procedure did not require the cognitive processing of number magnitude information at all, this may account for the fact that we did not observe any association of physiologically indicated threat / challenge and classification performance for incorrectly bisected triplets. This interpretation is in line with the results of Blascovich et al. (1999) who only found influence of threat / challenge on performance when the task at hand does require substantial cognitive processing, rather than being solvable by applying mere heuristics (such as the short-cut strategy of predominantly classifying a triplet as incorrectly bisected, based on the inclusion of sequences of adjacent numbers or steps of two, see above).

In sum, this suggests that the current manipulation of difficulty by means of higher demands on the processing of number magnitude information (in terms of larger bisection ranges) and smaller distances (to the correct mean of the triplet) may not have been the most fortunate choice. Instead, it might be interesting for future studies to manipulate numerical properties in a way that allow for an explicit differentiation of cognitive processing of number magnitude information and the application 
of heuristics or even fact-retrieval processes. Importantly, this can easily be achieved using variations of the NBT as demonstrated in previous studies (e.g., Nuerk et al., 2002; Moeller et al., 2009). For incorrectly bisected triplets, this would imply the manipulation of what was termed 'bisection possibility'. A triplet is only possible to be correctly bisected by an integer mean when the two outer numbers are of the same parity (i.e., odd or even, e.g., 23_25_29, correct mean would be 26). In contrast, there is no integer mean when the two outer numbers are of differing parity (e.g., 23_25_28, correct mean would be 25.5). Nuerk et al. (2002) observed a reliable effect of bisection possibility on both error rates and reaction times, with lower error rates and shorter reaction times for triplets possible to be bisected by an integer mean than for those not possible to be bisected by an integer mean. The authors attributed this to the application of a heuristic of using the fact that the two outer numbers differ in parity to indicate a classification of the respective triplet as incorrectly bisected. For correctly bisected triplets, on the other hand, Nuerk et al. (2002) manipulated whether the triplet is part of a multiplication table (e.g., 12_15_18) or not (e.g., 11_14_17). They found that triplets that are part of a multiplication table were classified faster as being correctly bisected than those not being part of a multiplication table. Nuerk et al. (2002) argued that concomitantly activated multiplication fact knowledge facilitated the classification as being correctly bisected.

Transferred to the present case of physiological indices of threat / challenge in the NBT, we would predict that the association between cardiovascular reactivity and numerical performance should be more pronounced for those conditions that require an explicit processing of number magnitude information and that cannot be assisted by heuristics or fact retrieval processes: correctly bisected triplets not part of a multiplication table, and incorrectly bisected triplets not possible to be bisected by an integer mean. This seems an interesting next step for future research to address, which would allow for more conclusive insights under which specific conditions a threat (rather than challenge) response to a numerical task comes with worse performance.

Taken together, in the current study we observed that individuals differentially respond to a numerical task - such as the NBT - with specific cardiovascular reactivity and that this physiological response was predictive of task performance. In line with the propositions of BPSM (e.g., Blascovich, 2008; Blascovich \& Mendes, 2010; Blascovich \& Tomaka, 1996) experiencing the NBT as a threat 
(rather than challenge), as reflected by cardiovascular markers on the physiological level, was associated with worse individual performance. Yet the data did corroborate the second assumption that task difficulty should moderate this association. Nevertheless, following up on these promising results, future research on the preconditions and consequences of this association of physiological responses and numerical performance would be desirable to explore the specific mechanisms - that is, the appraisal of personal skills and task demands - which contribute to this association. In the long run, this may allow for new possibilities when thinking of interventions specifically targeted at overcoming threat reactions to numerical tasks. 


\section{APPENDIX}

\section{Glossary of cardiovascular indicators:}

- $\quad$ Cardiac output $(\mathrm{CO})=$ cardiac performance $($ the higher $=$ the more challenged $)$

- $\quad$ Total Peripheral Resistance (TPR) = efficiency of mobilizing and transporting energy (the lower $=$ the more challenged)

- $\quad$ Threat-challenge index $=\mathrm{TCI}=\mathrm{zCO}-\mathrm{zTPR}($ the higher $=$ the more challenged $)$

- $\quad$ Heart Rate $(H R)=$ indicator for task engagement (higher than baseline = engaged)

- $\quad$ Pre-ejection period $($ PEP) $=$ ventricular contractility; indicator for task engagement (lower than baseline $=$ engaged) 


\section{FOOTNOTES}

1: The original sample included a total of twenty-six participants. However, due to technical problems, the complete set of physiological measures was only assessed for the reported sixteen participants.

2: As additional, exploratory measures, we also assessed self-reported demands and resources with one item each (demands: "To what extent do you experience the task as threatening?", resources: "To what extent do you believe you can effectively deal with the task?", 1-not at all to 7-compelety; cf. Tomaka et al., 1993). Auxiliary analyses demonstrated that self-reported resources tended to predict errors for small distance triplets, $r(16)=-.43, p=.094$, and large distance triplets, $r(16)=-.42$, $p=.103$, but not other performance indicators or CV indicators (all $p \mathrm{~s}>.165$ ). Similarly, self-reported demands tended to predict TPR reactivity, $r(16)=.44, p=.088$, as well as the number of errors for small bisection range, $r(16)=.70, p=.003$, and wide bisection range, $r(16)=.66, p=.005$ (but not the other indicators, all $p \mathrm{~s}>$.206). In sum, these correlations are - potentially due to statistical power not significant, but descriptively in the expected direction.

3: Although we did find evidence for task engagement from decreased levels of PEP during the task, an increase in HR, which typically accompanies decreased threat to indicate engagement, was absent. An explanation for this finding might be found in the type of task that we used here, which is somewhat less metabolically-demanding than, for instance, a speech task, for which both significantly decreased PEP and increased HR are typically found (e.g., de Wit et al., 2012). However, PEP is the most direct cardiovascular index of sympathetic influences on the cardiovascular system (Brownley, Hurwitz, \& Schneiderman, 2000). As sympathetic activation is the central process underlying task engagement, we are confident that in the current situation, sufficient signs of engagement are present to warrant an analysis of challenge and threat following the biopsychosocial model.

4: Note that in our analyses, we focused on number of errors as an indicator of classification performance, as reaction times in this study may not represent a valid performance indicator due to the implemented time limit ( 5 seconds) for each trial. 


\section{REFERENCES}

Ansari, T. L., \& Derakshan, N. (2011). The neural correlates of cognitive effort in anxiety: Effects on processing efficiency. Biological Psychology, 86(3), 337-348. doi:10.1016/j.biopsycho.2010.12.013

Ashcraft, M. (2002). Math Anxiety: Personal, Educational, and Cognitive Consequences. Current Directions In Psychological Science (Wiley-Blackwell), 11(5), 181-185.

Ashcraft, M. H., \& Kirk, E. P. (2001). The relationships among working memory, math anxiety, and performance. Journal of experimental psychology: General, 130(2), 224.

Baddeley, A. D. (1986). Working memory. Oxford: Oxford University Press.

Beilock, S. L. (2008). Math performance in stressful situations. Current Directions in Psychological Science, 17, 339--343.

Beilock, S. L. \& Carr, T. H. (2005). When high-powered people fail: Working memory and "choking under pressure" in math. Psychological Science, 16, 101-105.

Blascovich, J. (2000). Psychophysiological methods. In H. T. Reis, H. Judd, \& C. M. Judd (Eds.), Handbook of research methods in social psychology (pp. 117-137). Cambridge UK: Cambridge University Press.

Blascovich, J. (2008). Challenge, threat, and health. In J. Y. Shah, \& W. L. Gardner (Eds.), Handbook of motivation science (pp. 481-493). New York: Guilford Press.

Blascovich, J., \& Mendes, W. B. (2000). Challenge and threat appraisals: The role of affective cues. In J. Forgas (Ed.), Feeling and thinking: The role of affect in social cognition (pp. 59-82). Cambridge, UK: Cambridge University Press.

Blascovich, J., \& Mendes, W. B. (2010). Social psychophysiology and embodiment. In D. Gilbert, S. Fiske, \& G. Lindzey (Eds.), Handbook of social psychology (pp. 194-227). (5th Edition). New York: Wiley.

Blascovich, J., Mendes, W. B., Hunter, S. B., \& Salomon, K. (1999). Social “facilitation” as challenge and threat. Journal of Personality and Social Psychology, 31, 422-429.

Blascovich, J., Seery, M. D., Mugridge, C. A., Norris, R. K., \& Weisbuch, M. (2004). Predicting athletic performance from cardiovascular indexes of challenge and threat. Journal of 
Experimental Social Psychology, 40, 683-688.

Blascovich, J., \& Tomaka, J. (1996). The biopsychosocial model of arousal regulation. In M. Zanna (Ed.), Advances in experimental social psychology, Vol. 28. (pp. 1-51)New York: Academic Press.

Brownley, K. A., Hurwitz, B. E., \& Schneiderman, N. (2000). Cardiovascular psychophysiology. In J. T. Cacioppo, L. G. Tassinary, and G. G. Berntson (Eds.), Handbook of psychophysiology (pp. 224-264). New York: Cambridge University Press.

Cates, G. L., \& Rhymer, K. N. (2003). Examining the relationship between mathematics anxiety and mathematics performance: An instructional hierarchy perspective. Journal of Behavioral Education, 12(1), 23-34.

Derakshan, N., \& Eysenck, M. W. (2009). Anxiety, processing efficiency, and cognitive performance: New developments from attentional control theory. European Psychologist, 14(2), 168-176. doi:10.1027/1016-9040.14.2.168

De Wit, F. C., Scheepers, D., \& Jehn, K. A. (2012). Cardiovascular reactivity and resistance to opposing viewpoints during intragroup conflict. Psychophysiology, 49(11), 1691-1699.

Eden, C., Heine, A., \& Jacobs, A. M. (2013). Mathematics Anxiety and Its Development in the Course of Formal Schooling-A Review. Psychology, 4(06), 27.

Else-Quest, N. M., Hyde, J. S., Linn, M. C. (2010). Cross-national patterns of gender differences in mathematics: A meta-analysis. Psychological Bulletin, 136, 103-127.

Eysenck, M. W., Derakshan, N., Santos, R., \& Calvo, M. G. (2007). Anxiety and cognitive performance: Attentional control theory. Emotion, 7(2), 336-353. doi:10.1037/15283542.7.2.336

Gratton, G., Coles, M. G., \& Donchin, E. (1992). Optimizing the use of information: strategic control of activation of responses. Journal of Experimental Psychology: General, 121(4), 480.

Gross, J., Hudson, C. and Price, D. (2009). The Long Term Costs of Numeracy Difficulties. Every Child a Chance Trust and KPMG: London.

Hopko, D. R., Ashcraft, M. H., Gute, J., Ruggiero, K. J., \& Lewis, C. (1998). Mathematics anxiety and working memory: Support for the existence of a deficient inhibition mechanism. Journal of 
Anxiety Disorders, 12, 343-355.

Hopko, D. R., McNeil, D. W., Lejuez, C. W., Ashcraft, M. H., Eifert, G. H., \& Riel, J. (2003). The effects of anxious responding on arithmetic and lexical decision task performance. Journal of Anxiety Disorders, 17, 647-665.

Huber, S., Moeller, K., Nuerk, H.-C., Macizo, P., Herrera, A., \& Willmes, K. (2013). Cognitive control in number processing - A computational model. In R. West \& T. Stewart (Eds.), Proceedings of the 12th International Conference on Cognitive Modeling (pp. 185-190). Ottawa: Carleton University.

Krinzinger, H., Kaufman, L., \& Willmes, K. (2009). Math anxiety and math ability in early primary school years. Journal of Psycho-educational Assessment, 27, 206-225.

Macizo, P., \& Herrera, A. (2011). Cognitive control in number processing: Evidence from the unitdecade compatibility effect. Acta Psychologica, 136, 112-118.

Maloney, E. A., \& Beilock, S. L. (2012). Math anxiety: Who has it, why it develops, and how to guard against it. Trends In Cognitive Sciences, 16(8), 404-406. doi:10.1016/j.tics.2012.06.008

Moeller, K., Fischer, M. H., Nuerk, H., \& Willmes, K. (2009). Eye fixation behaviour in the number bisection task: Evidence for temporal specificity. Acta Psychologica, 131, 209-220. doi:10.1016/j.actpsy.2009.05.005

Moenikia, M., \& Zahed-Babelan, A. (2010). A study of simple and multiple relations between mathematics attitude, academic motivation and intelligence quotient with mathematics achievement. Procedia - Social and Behavioral Sciences, 2, 1537-1542.

Moore, L. J., Vine, S. J., Wilson, M. R., \& Freeman, P. (2012).The effect of challenge and threat states on performance: An examination of potential mechanisms. Psychophysiology, 49, 14171425.

Nisbett, R. E., \& Wilson, T. D. (1977). Telling more than we can know: Verbal reports on mental processes. Psychological Review, 84, 231-259.

Nuerk, H.-C., Geppert, B. E., van Herten, M. \& Willmes K. (2002). On the impact of different number representations in the number bisection task. Cortex, 38, 691-715.

Parsons, S. \& Bynner, J. (2005). Does numeracy matter more? NRDC (National Research and 
Development Centre for adult literacy and numeracy).

Ramirez, G., Gunderson, E. A., Levine, S. C., \& Beilock, S. L. (2013). Math anxiety, working memory and math achievement in early elementary school. Journal of Cognition and Development, 14, 187-202.

Scheepers, D., de Wit, F., Ellemers, N., \& Sassenberg, K. (2012). Social power makes the heart work more efficiently: Evidence from cardiovascular markers of challenge and threat. Journal of Experimental Social Psychology, 48(1), 371-374. doi:10.1016/j.jesp.2011.06.014

Seery, M. D., Weisbuch, M., Hetenyi, M. A., \& Blascovich, J. (2010). Cardiovascular measures independently predict performance in a university course. Psychophysiology, 47(3), 535-539.

Sherwood, A., Allen, M. T., Fahrenberg, J., Kelsey, R. M., Lovallo, W. R., \& van Dooren, L. J. P. (1990). Methodological guidelines for impedance cardiography. Psychophysiology, 27, 1-23.

Núñez Peña, M. I., \& Colomé, À. (2014). Reactive Recruitment of Attentional Control in Math Anxiety: An ERP Study of Numeric Conflict Monitoring and Adaptation. PLoS One, 2014, vol. 9, num. 6, p. e99579.

Tomaka, J., Blascovich, J., Kelsey, R. M., \& Leitten, C. L. (1993). Subjective, physiological, and behavioral effects of threat and challenge appraisal. Journal of Personality and Social Psychology, 18, 616-624. doi: 10.1037/0022-3514.65.2.248

Tzelgov, J., Henik, A., \& Berger, J. (1992). Controlling Stroop effects by manipulating expectations for color words. Memory \& Cognition, 20, 727-735.

Weisbuch-Remington, M., Mendes, W. B., Seery, M. D., \& Blascovich, J. (2005). The influence of religious stimuli outside of subjective awareness during motivated performance situations. Personality and Social Psychology Bulletin, 31, 1203-1216.

Wood, G., Nuerk, H.-C., Moeller, K., Geppert, B., Schnitker, R., Weber, J., et al. (2008). All for one but not one for all: How multiple number representations are recruited in one numerical task. Brain Research, 1187, 154-166. 